\title{
SMALL CRATERS AND THEIR DIAGNOSTIC POTENTIAL
}

\author{
R. Bugiolacchi ${ }^{\mathrm{a}, \mathrm{b}}$ \\ ${ }^{a}$ Space Science Laboratory, MUST, Avenida Wai Long, Taipa, Macau - rbugiolacchi@ must.edu.mo \\ ${ }^{\text {b }}$ Dept. of Earth Sciences, University College London, Gower Street, WC1E 6BT, London, UK - roberto.bugiolacchi@ucl.ac.uk
}

KEY WORDS: Moon, Lunar science, Tycho crater, cratering, geochronology, geomorphology

\begin{abstract}
:
I analysed and compared the size-frequency distributions of craters in the Apollo 17 landing region, comprising of six mare terrains with varying morphologies and cratering characteristics, along with three other regions allegedly affected by the same secondary event (Tycho secondary surge). I propose that for the smaller crater sizes (in this work 9-30 m), a] an exponential curve of power $0.18 \mathrm{D}$ can approximate $\mathrm{Nkm}^{-2}$ crater densities in a regime of equilibrium, while b] a power function $\mathrm{D}^{-3}$ closely describes the factorised representation of craters by size $(1 \mathrm{~m})$. The saturation level within the Central Area suggests that c] either the modelled rates of crater erosion on the Moon should be revised, or that the Tycho event occurred much earlier in time than the current estimate. We propose that d] the size-frequency distribution of small secondary craters may bear the signature (in terms of sizefrequency distribution of debris/surge) of the source impact and that this observation should be tested further.
\end{abstract}

\section{INTRODUCTION}

When two planetary bodies collide the energy of impact is recorded as material displacement at the point of contact (unless there is mutual annihilation), creating both negative (a crater) and positive surface expressions (rim and peaks), plus scattered debris (ejecta). Each impact structure has a finite 'lifespan' due to entropic processes leading to disaggregation of the raised structures (by space weathering and thermal expansion) and infill of the excavation (gravity-led transportation and ballistics). The rate of disintegration is size-dependent and governed by several physical factors, such as the cohesion and porosity of the target materials (regolith thickness/bedrock), terrain slope, direct modification or destruction by larger impacts (Melosh, 2011).

Production function models for interplanetary bodies and their likelihood of impact with time are well constrained for the last $\sim 3 \mathrm{Ba}$ (Neukum et al., 2001). Craters around $100 \mathrm{~m}$ in diameter on the Moon are produced by celestial rocky materials of mass $\sim 4 \times 10^{5} \mathrm{~kg}$ and diameter $\sim 6 \mathrm{~m}(\sim 10 \mathrm{~m}$ if cometary) (Holsapple, 1993). Since a body of this size would still produce a $\sim 70 \mathrm{~m}$ crater on Earth, it is important to further constrain their present flux.

\subsection{Aims}

By looking at a lunar region that has been mechanically resurfaced within recent geological times $(\sim 110 \mathrm{Ma}$, Arvidson et al., 1976) we can in principle investigate the distribution and rate of decay of small impacts in relation to their size across a representative sample of terrain types. We are seeking possible crater size frequency distribution patterns in the smallest sizes $(\sim 10-100 \mathrm{~m})$ against a theoretical stochastic distribution both within the unequivocally affected areas, such as Tycho's bright rays (NACs *808RE-*186LE, Fig. 1), to alleged distal landings, as in this study the explored (A17) Taurus-Littrow Valley.

\section{DATA AND METHOD}

Fig. 1 upper inset (A17) shows the region of this investigation and the unit boundaries drawn from observation of variations in surface texture, 'qualitative' cratering distribution, and albedo. Nomenclatures used in the text are: Mare Old ('unaffected' mare), MO; Light Mantle 'North', LM_n; Light Mantle 'South', LM_s; and Light Mantle, LM; and Uplands (Massifs tops), 'UP'.

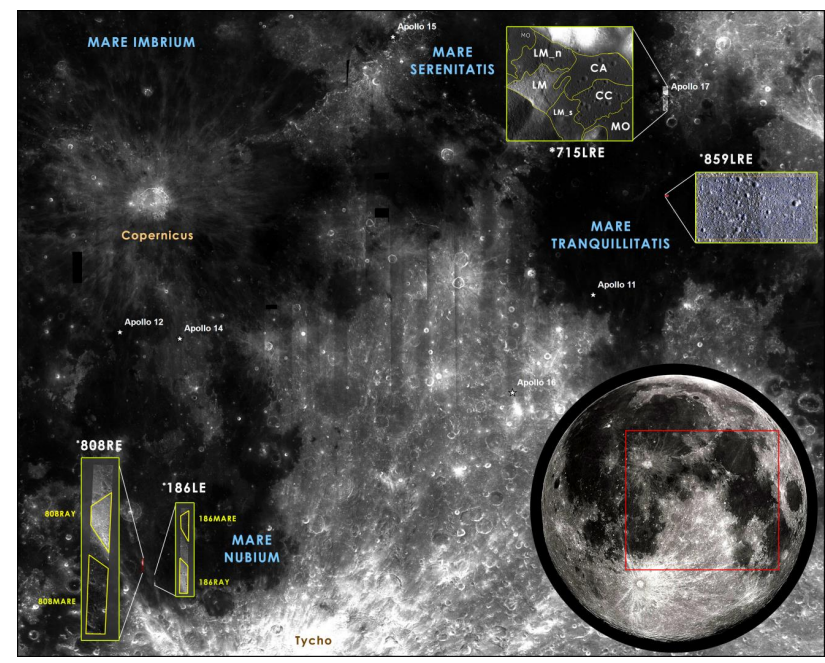

Figure 1. Locations of areas under investigation. NAC images (shortened product name - Robinson et al., 2005) vary both in ground resolution $(0.5-1.5 \mathrm{~m} / \mathrm{px}-* 186 \mathrm{LE}-* 715 \mathrm{LRE})$ and illumination conditions (incidence angle 31-72 deg. - *808RE*859LRE). See APPENDIX for full size image

A comprehensive and multiple crater survey was carried out on a NAC image pair (M104311715LRE) with $1.5 \mathrm{~m} / \mathrm{px}$ resolution by the author using different magnification levels and starting from different area points each time to minimise human bias. ArcGIS was used to mark craters using standard drawing tools. The results were then compared with those generated by citizen scientists and, in a subset, by a team of experts (Bugiolacchi et al., 2016).

A similar approach, bar the citizen scientists input, was employed to survey craters distribution astride a prominent Tycho ray within the southern Mare Nubium using two higher resolution images (NAC M111722186LE-M1180249808RE, 
0.5-0.8 m/px, see fig. 1). This time the add-on to ArcGIS CraterTools (Kneissl et al., 2011) was used for the crater count. Finally, a forth sampling region was selected in Mare Tranquillitatis to serve as a random 'mare background' but still related to the Apollo 17 region mare infill (NAC M1096293859LRE, res. $1.2 \mathrm{~m} / \mathrm{px}$ ).

Over 90,000 craters were marked ranging from 4 meter diameter upwards. However, given the threefold variation in resolution between images $(0.5$ to $1.5 \mathrm{~m} / \mathrm{px})$, diameters $>9$ meters were set as lower limit to minimise marking precision uncertainties and fair comparison.

Crater histograms were produced using $1 \mathrm{~m}$ bins and sizes up to $30 \mathrm{~m}$ were investigated in detail (Fig. 2). It is worth emphasising that this size range represents on average over $90 \%$ (median 92\%) of all craters between 10 and $100 \mathrm{~m}$ and no bin size is larger than $1 \%$ above $30 \mathrm{~m}$. 'A' represents the number of craters per sq. $\mathrm{km}$ for each bin size. ' $\mathrm{B}$ ' is derived from the ratio between adjacent bins and used to emphasise similar patterns in number representation between geographical regions. ' $C$ ' factors the bin size between $10-100 \mathrm{~m}$ to unity $(100, \%)$. ' $\mathrm{D}$ ' plots all craters $>30 \mathrm{~m}$ in cumulative form with pseudo-log binning (Michael and Neukum, 2010), giving the relative small sample size (revealed by the large sigma delta error bars).

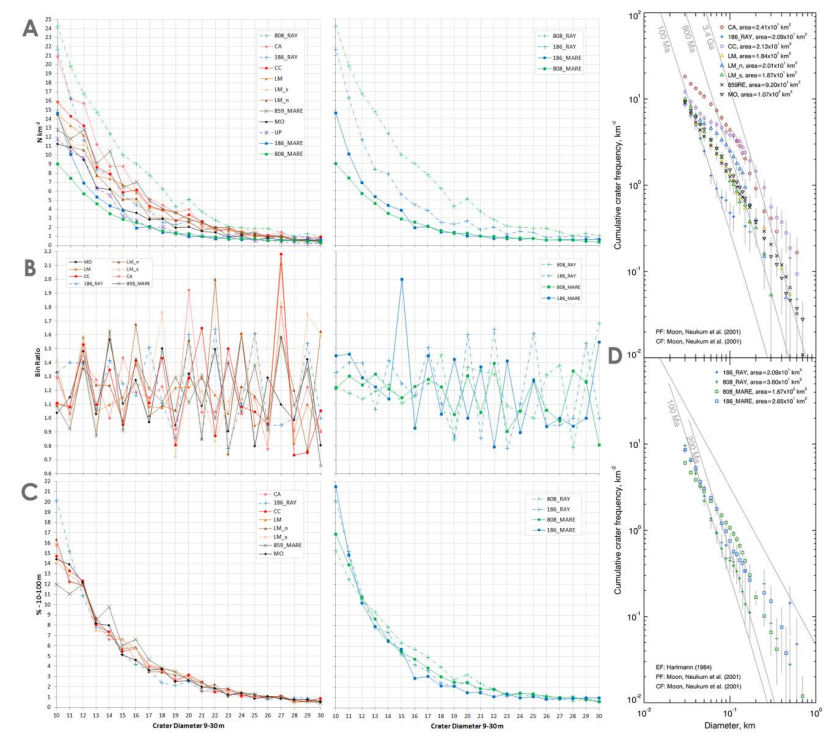

Figure 2. A - histogram of number of craters per $1 \mathrm{~m}$ bin size per $\mathrm{km}^{-2}$ (i.e., density); $\mathrm{B}$ - ratio between adjacent $\mathrm{Nkm}^{-2}$ bins; $\mathrm{C}$ - bin sizes 10 to $100 \mathrm{~m}$ factorised to unit, in this case 100 to give percentage; $\mathrm{D}-$ pseudo-log binned histogram for sizes $>30$ m using CraterstatsII (Michael and Neukum, 2010). Each data point is not fit to isochrones, which are drawn for reference only. See APPENDIX for full size image

\section{RESULTS}

Crater density comparison between units within the TaurusLittrow valley and the other three sample locations shows predictable differences but also unexpected correlations. The highest densities of craters $<\sim 30 \mathrm{~m}$ are found across the heavily cratered centre part of the region (CA and $\mathrm{CC}$ ) and the alleged (Arvidson et al., 1976) coeval ray (808_ and 186_ rays, Fig. 2A), with densities up to $21-24 \mathrm{Nkm}^{-2}$ for CA and the Nubium ray (9-10 $\mathrm{m}$ craters). The crater-density for the mare outside the high albedo rays (Mare Nubium) is lower than all the sampled areas (808_ and 186_Mare) including Mare Tranquillitatis (859_Mare) and MO. However, apart from 808_MARE, the crater density for all the remaining units follows a general exponential curve approximated by the equation:

$$
\mathrm{C}_{d}=85 \times 10^{-0.18 D}
$$

where $\quad \mathrm{C}_{d}=$ crater density $\left(\mathrm{Nkm}^{-2}\right)$

$$
D=\text { crater diameter (meters) }
$$

Fig. 2A hints at 'oscillations' in crater bin sizes, amplified in Fig. 2B where adjacent bin ratios $\left(\mathrm{Nkm}^{-2}\right)$ appear to occur at certain size points, in particular at even numbers $(12,14,16 \mathrm{~m}$ etc.) except for $27 \mathrm{~m}$. This trend is absent or subdued on the Nubium ray where only the surge portion of NAC $* 186$ shows some correlation (at 12, 14, 20, $22 \mathrm{~m}$ ).

To compare further the $10-100 \mathrm{~m}$ range size, we have normalised to unity $(100, \%)$, which means that for instance, in the Taurus-Littrow (A17) mare region and Mare Tranquillitatis (Fig. 2C) $12 \%$ of all craters are between 11 and $12 \mathrm{~m}$ in size (left graph), while in Mare Nubium the same sizes are between 10 and $11 \%$ (right graph).

The larger craters population could not be compared directly since the A17 region has numerous $>500 \mathrm{~m}$ impacts, while the higher resolution but smaller areas, do not have a significant crater population $>100 \mathrm{~m}$ in size, also due to the images resolution and area. Nonetheless, we have plotted the data using the standard CratersStatsII platform (Kneissl et al., 2011) to gain further information from compararing units' slopes (Fig. 2D). We did not use the fit alghorythms available since no meaningful age estimation, AMA (Absolute Model Age), could be extracted. However, we have plotted the production functions that best approximate limited ranges for each unit, such as $100 \mathrm{Ma}$ for 186_RAY, $800 \mathrm{Ma}$ for several A17 units and Mare Tranquillitatis (859RE), and $3.7 \mathrm{Ga}$ for mare areas MO and 859RE. No 'cumulative functions', such as 'resurfacing', were engaged.

\section{DISCUSSION}

The histograms show that the distribution of small craters in the A17 region $(\leq 30 \mathrm{~m})$ was significantly modified by the arrival of a secondary surge, alleged to belong to the distal Tycho impact. In the denser area of the surge (CA), we see the sizefrequency distribution following an exponential trend that could reflect a saturation level for the lunar maria, at least in the sub$30 \mathrm{~km}$ crater range (as described by eq. (1)). Moving away from this area, and arguably from the epicentre of the surge, we notice a gradual shift towards a 'background-type' distribution. Plotted together with the other sampled areas the average density decreases in this sequence: 808 RAY $\rightarrow \boldsymbol{C A} \rightarrow 186 \mathrm{RAY} \rightarrow \boldsymbol{C C} \rightarrow \boldsymbol{L} \boldsymbol{M} \rightarrow \boldsymbol{L M} \_\boldsymbol{s} \rightarrow \boldsymbol{L} \boldsymbol{M} \_\boldsymbol{n} \rightarrow 85$ 9_MARE $\rightarrow \boldsymbol{M O} \rightarrow \boldsymbol{U} \boldsymbol{P} \rightarrow 186$ MARE $\rightarrow$ 808_MARE (Fig. 2A), reflecting a gradually smaller contribution from secondaries. The small craters distribution atop the massifs (UP) and the maria (MO) is nearly identical (at this range size), suggesting an equilibrium in their production level (and described by eq.s (1) and (2)), possibly applicable moon-wide, an hypothesis needed to be tested further.

Based on the straight relationship formulated by Basilevsky (1976), $\mathrm{T}_{\mathrm{Ma}}=2.5^{*} D$, ( $\mathrm{T}_{\mathrm{MA}}$ is time in $\mathrm{Ma}$ and $D$ is crater diameter in meters) the small secondary craters excavated by the surge should all but eroded long ago ( 50 Ma lifetime for the 
$20 \mathrm{~m}$ fraction, or $\sim 70 \mathrm{Ma}$, Fassett and Thomson, 2014). Given that the event is estimated at $\sim 110$ Ma (Arvidson et al. 1976), the crater count should have approached the background level by now. This discrepancy raises questions on either the erosion rate models or the age of the Tycho event itself. However, when the relative bin size representation is computed (Fig. 2C), compensating for bias effects introduced by the difference in sampled area, we see a remarkable agreement between crater sizes, for instance in all but one unit (186_MARE) the 11-13 m craters representation stands at $20 \%$ (18\% for the odd one). Since the represented regions are rather remote and the craters marked using different images with different characteristics (illumination, resolution, and area), it appears that the relative crater size representation displays a relative saturation threshold with a ' -3 ' power curve:

$$
\%_{R}=2 \times 10^{5} \cdot D^{-3}
$$

where

$$
\begin{aligned}
& \%_{R}=\text { relative crater density }(\%) \\
& D=\text { crater diameter (meters) }
\end{aligned}
$$

Last, though no least, looking at the data in Fig.s 2A and 2B it emerges that most crater distributions affected by the surge share some common characteristics in the size-frequency distribution, such as the 'hump' at the $12 \mathrm{~m}$ bin. Fig. $2 \mathrm{C}$ instead highlights a similar trend between crater sizes 18-22 $\mathrm{m}$ for the 808_, 186_RAY, CC, and CA.

Is this characteristic to Tycho, i.e., did the ejecta surge carry size-sorted debris? Do other secondary surges (rays) show similar distributions unique to each primary event? Thus, could this trend represent an identifying signature, a kind of impact event fingerprint? Alternatively, the crater size-frequency distribution at the smaller crater sizes might be influenced by post-impact factors, such as regolith depth, surge porosity/density, or even the way craters are affected to lunar moonquakes/shakes differently in accordance to the frequency of the tremors.

\section{CONCLUSIONS}

Following the analysis of size-frequency distribution of craters in a highly heterogeneous and well-studied region of the Moon, together with three other related sites, we propose that for the smaller crater sizes (in this work 9-30 m), a] an exponential curve of power $-0.18 D$ (eq. (1)) can approximate $\mathrm{Nkm}^{-2}$ crater densities in a regime of equilibrium, while $\mathbf{b}$ ] a power function $D^{-3}$ (eq. (2)) closely describes the factorised representation of craters by size.

The saturation level within the Central Area (CA, Fig. 1) suggests that $\mathbf{c}$ ] either the modelled rates of crater erosion on the Moon should be revised, or that the Tycho event is younger than the accepted estimate.

We propose that d] the size-frequency distribution of small secondary craters may bear the signature (in terms of sizefrequency distribution of debris/surge) of the source impact and this observation should be tested further.

\section{REFERENCES}

Arvidson, R., Drozd, R., Guinness, E., Hohenberg, C., Morgan, C., Morrison, R., Oberbeck, V., 1976. Cosmic ray exposure ages of Apollo 17 samples and the age of Tycho. Proc. Lunar Planet. Sci. Conf. 7th, 2817-2832.Basilevsky, A.T., 1976. Lunar and Planet. Sci. Conf. Proc. pp. 1005- 1020.

Bugiolacchi, R., Bamford, S., Tar, P., Thacker, N., Crawford, I.A., Joy, K.H., Grindrod, P.M., Lintott, C., 2016. The Moon Zoo citizen science project: Preliminary results for the Apollo 17 landing site. Icarus 271, 30-48.

Fassett, C.I., Thomson, B.J., 2014. Crater degradation on the lunar maria: Topographic diffusion and the rate of erosion on the Moon. J. Geophys. Res. Planets 119, 2255-2271.

Holsapple K.A. 1993. The scaling of impact processes in planetary sciences. Ann. Rev. of Earth and Planet. Sci. 21, 333373.

Kneissl T., van Gasselt, S., and Neukum, G. 2011. Mapprojection-independent crater size-frequency determination in GIS environments. New software tool for ArcGIS. Planet. Space Sci. 59, 1243-1254.

Michael, G.G., Neukum, G., 2010. Planetary surface dating from crater size-frequency distribution measurements: Partial resurfacing events and statistical age uncertainty. Earth Planet. Sci. Lett. 294, 223-229.

Melosh, H.J. 2011. Planetary surface processes. Cambridge Uni. Press. ISBN 9780521514187

Neukum, G., Ivanov, B.A., Hartmann, W.K., 2001. Cratering records in the inner solar system in relation to the lunar reference system. Space Sci. Rev. 96, 55-86.

Robinson, M. S., Eliason, E. M., Hiesinger, H., Jolliff, B. L., McEwen, A. S., Malin, M. C., Ravine, M. A., Roberts, D., Thomas, P. C., Turtle, E. P., 2005. LROC -- Lunar Reconnaissance Orbiter Camera, in: Mackwell, S., Stansbery, E. (Eds.), 36th Annual Lunar and Planetary Science Conference, Lunar and Planetary Science Conference.

\section{ACKNOWLEDGEMENTS}

This study was supported by the Macau Science and Technology Development Fund 039/2013/A2 


\section{APPENDIX. FULL SIZE FIGURES}

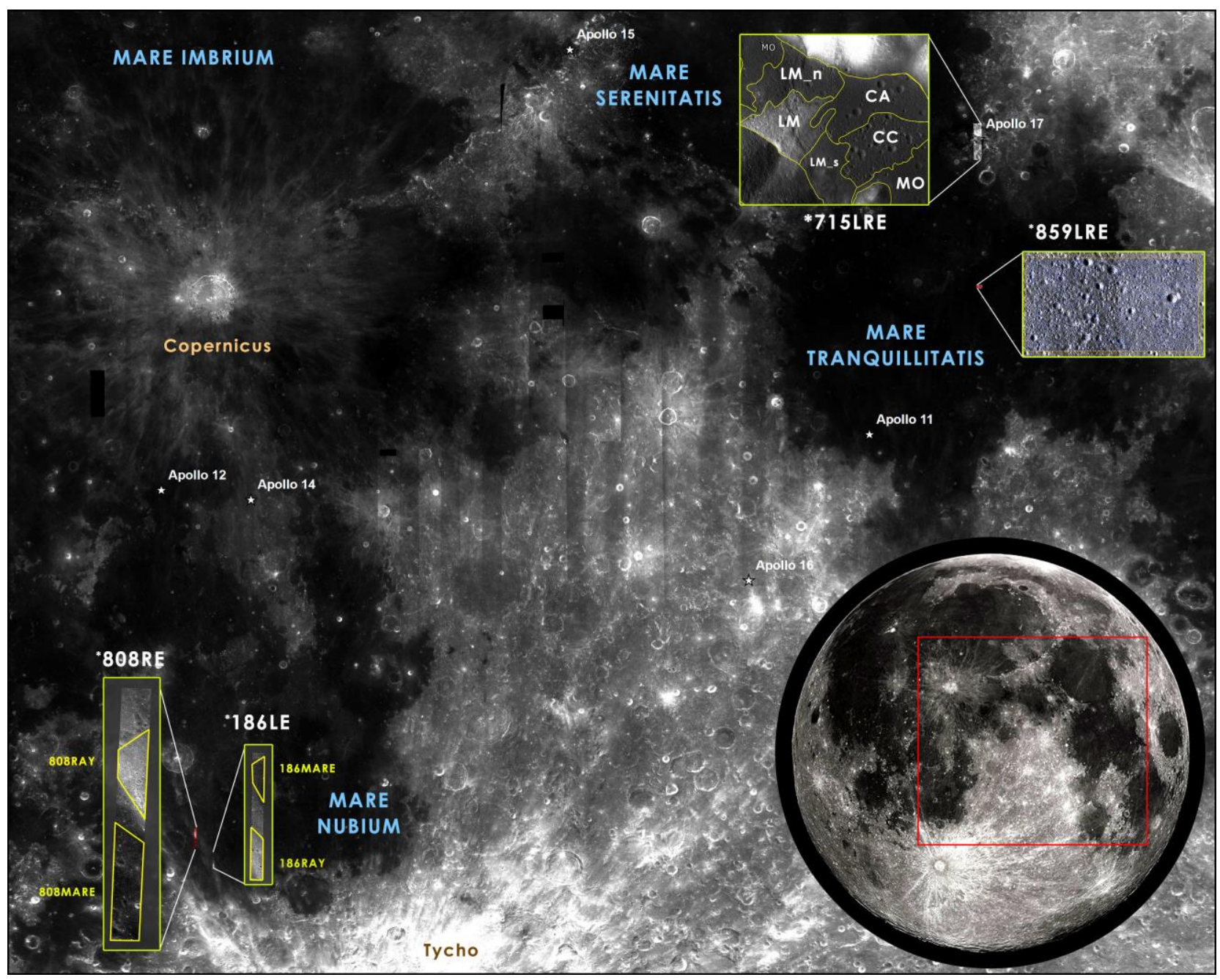

Figure 1. Locations of areas under investigation. NAC images (shortened product name - Robinson et al., 2005) vary both in ground resolution (0.5-1.5 m/px - *186LE-*715LRE) and illumination conditions (incidence angle 31-72 deg. - *808RE-*859LRE). 

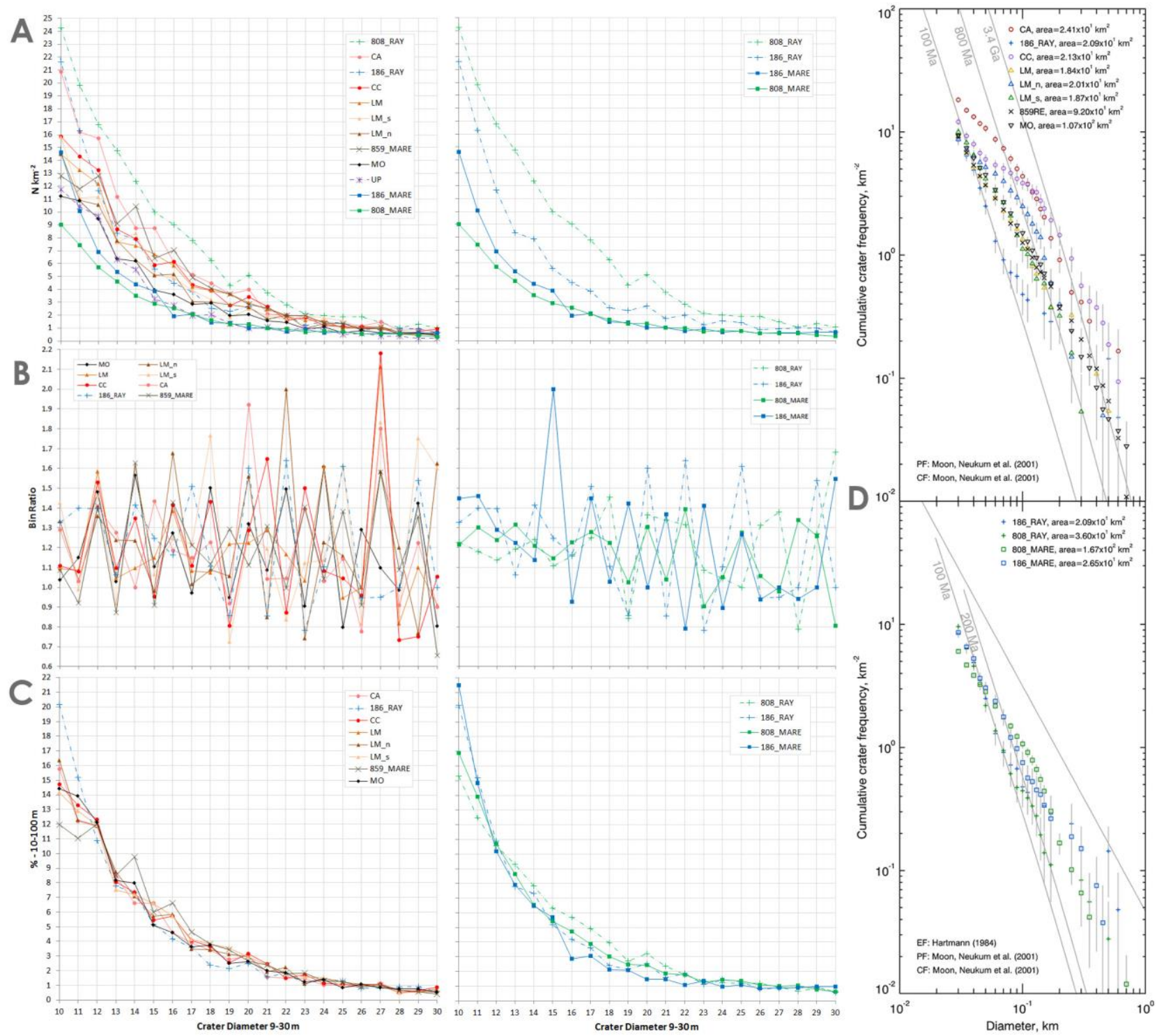

Figure 2. A - histogram of number of craters per $1 \mathrm{~m}$ bin size per $\mathrm{km}^{-2}$ (i.e., density); B - ratio between adjacent $\mathrm{Nkm}^{-2}$ bins; $\mathrm{C}-$ bin sizes 10 to $100 \mathrm{~m}$ factorised to unit, in this case 100 to give percentage; D - pseudo-log binned histogram for sizes $>30 \mathrm{~m}$ using CraterstatsII (Michael and Neukum, 2010). Each data point is not fit to isochrones, which are drawn for reference only. 